\title{
A Slope-Adapted Sample-Tilting Method for Profile Measurement of Microstructures with Steep Surfaces
}

\author{
Hui Fang, Bin Xu, Wei Chen, Hairong Tang, and Shiping Zhao \\ School of Manufacturing Science and Engineering, Sichuan University, 24 South No. 1 Ring Road, Chengdu 610065, China \\ Correspondence should be addressed to Bin Xu; bin_xu@outlook.com
}

Received 29 January 2015; Revised 21 April 2015; Accepted 23 April 2015

Academic Editor: Yong Zhu

Copyright (C) 2015 Hui Fang et al. This is an open access article distributed under the Creative Commons Attribution License, which permits unrestricted use, distribution, and reproduction in any medium, provided the original work is properly cited.

\begin{abstract}
This paper presents a slope-adapted sample-tilting method for the profile measurement of microstructures with steep surfaces. Distinct from the traditional scanning method that has the restriction of a maximum detectable angle, this method corrects the sample-stylus relative angle during the measurement of the steep surface to eliminate the profile deviation and the scanning blind region. The performance of the proposed method was verified by simulations that measured the surface profiles of a trapezoidal microstructure and a spherical microstructure, finding maximum errors of $0.15 \mu \mathrm{m}$ and $1.71 \mu \mathrm{m}$, respectively, compared to $3.63 \mu \mathrm{m}$ and $7.85 \mu \mathrm{m}$ using the traditional scanning method. The proposed method enables accurate profile measurement and quality control of microstructures with steep surfaces.
\end{abstract}

\section{Introduction}

Precision microstructures with steep surfaces are widely used in precision industries and have recently become a trend in new generation manufacturing technology [1-4]. Such microstructures, as shown in Figure 1(a), including microlenses employed by Shack-Hartmann wavefront sensors, microprisms of a light guide plate in solar energy systems, and microgrooves in aerospace micropropulsion engines, typically have critical dimensions from several to several hundred microns and a maximum local slope of up to 90 degrees [5-9]. Accurate profile measurement is important because the geometric quality of the microstructure surfaces dominates the working performance of microstructure-based devices. The main difficulty in profiling microstructures with steep surfaces lies in the maximum detectable angle of the profiling system. In a stylus-based profiling system with a maximum detectable angle of 45 or 60 degrees $[4,10]$, the probing point tapers from the stylus tip to its flanks, resulting in the profile deviations shown in Figure 1(b). Using an optical profiling system, such as an autofocus profilometer or interferometer, with a maximum detectable angle of 15 or 30 degrees, it is difficult to achieve a useful reflection signal from the steep surface $[4,11]$.
Over the years, work has been conducted to resolve this issue. Ju et al. developed a novel scanning tunneling microscope to measure the surface profile of a microstructure with a 90-degree steep sidewall $[12,13]$. Marinello et al. combined the methods of controlled tilting and image processing to increase the maximum detectable slope of an optical profiler [14]. Cho et al. built a three-dimensional atomic force microscope (AFM) to measure the surface roughness of undercut and sidewall structures [15]. Pan et al. proposed a stitching double-tilt image method to measure sub-50 nm line widths employing an AFM system [16]. The above approaches manually tilt the sample and measure the sample repeatedly under different tilt angles, and the entire surface profile is reconstructed by a data fusion algorithm. However, the measurement process is time consuming, and the measurement errors introduced by the manual sampletilting and the data fusion algorithm are too strong to be eliminated by compensation. Henselmans et al. designed a noncontact measurement machine to scan the surface profile of freeform optics [17], and Weckenmann et al. proposed a sensor tilting method to enhance the measurement capability of microstructures $[18,19]$. These two methods appropriately tilt the probing sensor when scanning a steep surface. However, as the lateral resolution of the employed probes is limited 


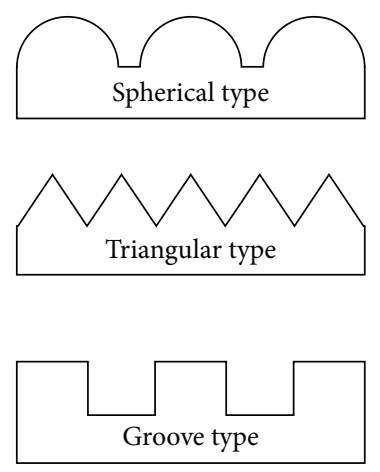

(a)

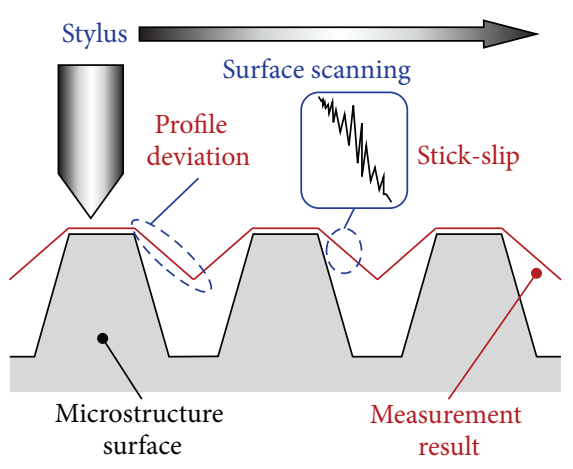

(b)

FIGURE 1: Stylus profiling microstructures. (a) Representative microstructures with steep surfaces. (b) Schematic of measurement deviation.

to the order of hundred microns, the application is unable to measure microstructures profiles with critical dimensions from several to several hundred microns. Although many measurement systems and methods have been established for the profile measurement of microstructures with steep surfaces [11, 20-24], a rapid, simple, and cost-effective measurement method is desired.

This paper presents a slope-adapted sample-tilting method for the profile measurement of microstructures with steep surfaces. First, a novel stylus-based profiling system that implements the proposed method is reported. Relevant theoretical work that underlies the realization of the system is described, including the principle of the proposed method and strategies to control the planar scanner and to tilt the sample. Simulations of the profiling of the steep surfaces of two microstructures were then carried out to verify the feasibility of the proposed method.

\section{Measurement System and Slope-Adapted Sample-Tilting Method}

Figure 2 shows the schematic of the stylus-based profiling system designed to implement the proposed method. The system consists of a displacement sensor with a microstylus as a $Z$-direction scanner, an $X$ motorized driven stage for planar scanning, and a rotating spindle to adjust the samplestylus relative angle. The system has a solid base plate and a bridge, both made of granite. The entire system is located on a vibration isolation table, so that the measurement would not be affected by external vibrations. The displacement sensor, which measures the axial motion of the microstylus in the $Z$-direction, is installed on the granite bridge by manual $X Y Z$ stages. The $X$ motorized driven stage with a sample is mounted on the center of the spindle rotary table. The surfaces of the $X$ motorized driven stage and the turntable are set parallel and perpendicular to the vibration-isolation table surface, respectively.

As shown in Figure 3, the slope-adapted sample-tilting method adjusts the sample-stylus relative angle during surface profile measurement. This method enables the contact

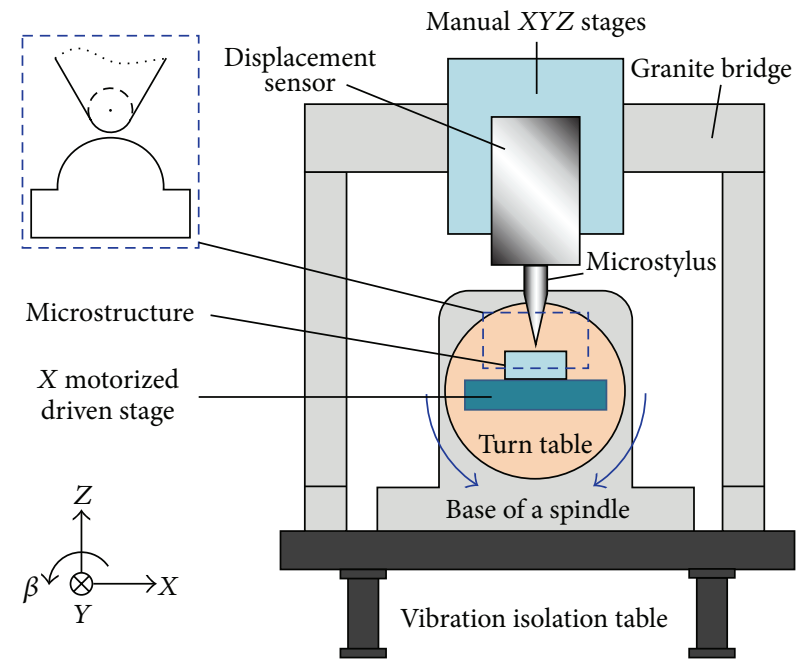

FIGURE 2: Schematic of the measurement system to implement the proposed method.

point to stay at the stylus tip and makes the profile measurement fulfill the requirement of the maximum detectable angle of the system, thereby eliminating the profile deviation.

Before measurement, the offset error of the sensor axis and the zero point error of the sensor output should be calibrated by testing a master cylinder [23]. After the calibration, the sensor output is the distance from the radius center of the stylus tip to the rotation axis of the spindle. As shown in Figure 4(a), the profile represented by the scanning results, $P_{i}^{\prime}$, is an envelope of the sample surface with a distance $r$, which is the tip radius of the stylus. The scanning results, $P_{i}^{\prime}$, are expressed as

$$
\left[\begin{array}{c}
P_{1}^{\prime} \\
P_{2}^{\prime} \\
P_{3}^{\prime} \\
\vdots \\
P_{i}^{\prime}
\end{array}\right]=\left[\begin{array}{cc}
P_{1-X}^{\prime} & P_{1-Z}^{\prime} \\
P_{2-X}^{\prime} & P_{2-Z}^{\prime} \\
P_{3-X}^{\prime} & P_{3-Z}^{\prime} \\
\vdots & \vdots \\
P_{i-X}^{\prime} & P_{i-Z}^{\prime}
\end{array}\right],
$$




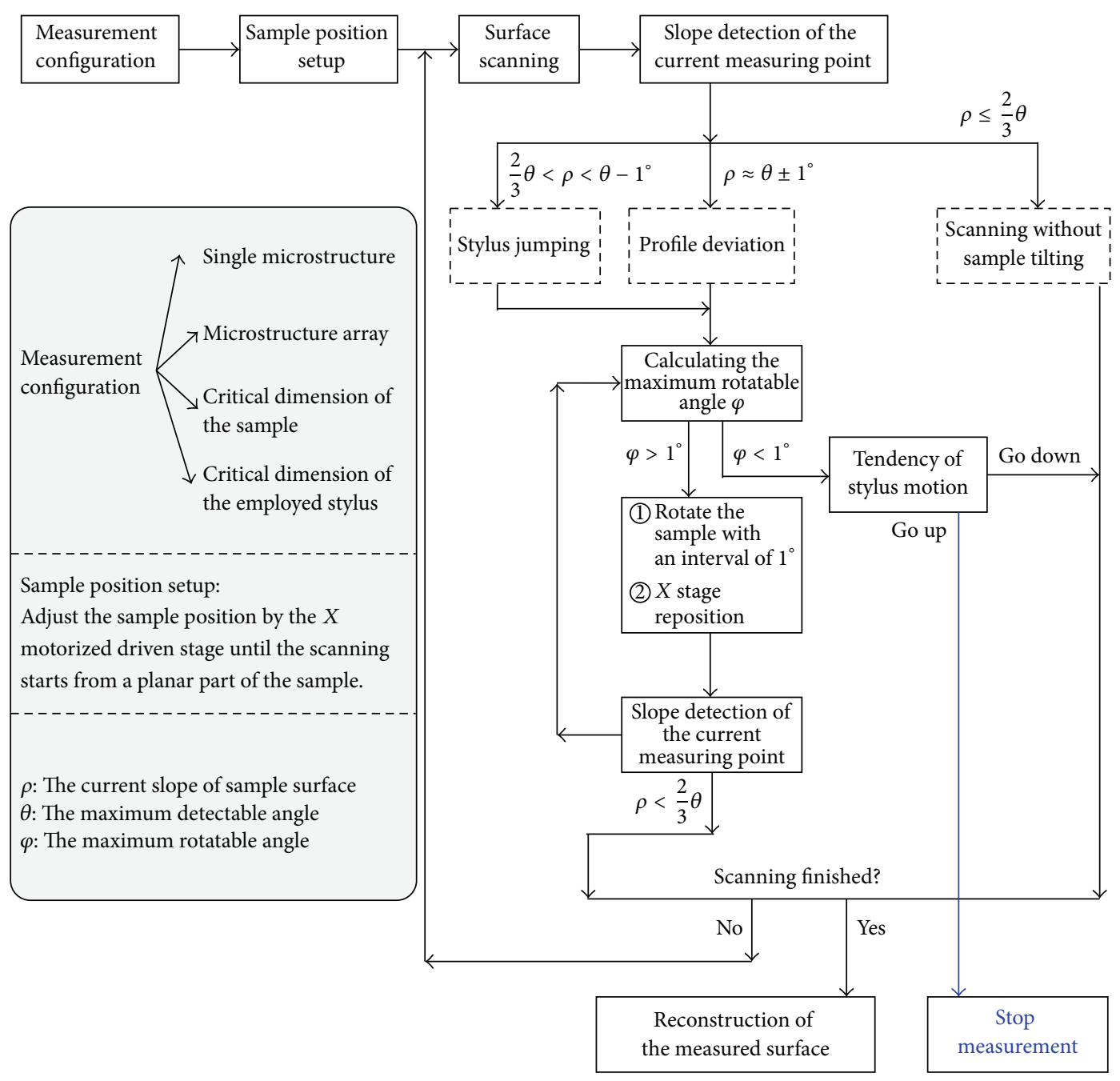

FIGURE 3: Flowchart of the slope-adapted sample-tilting method.

where $P_{i-X}^{\prime}$ is obtained from the $X$ motorized driven stage and $P_{i-Z}^{\prime}$ is the output of the displacement sensor.

The profile measurement starts from a flat part of the sample surface, and the scanning interval along the $X$ direction is assumed to be $\Delta x$. After a few points were measured, the local slope, $\rho_{i}$, is detected for the current measuring point $P_{i}$. Then, $\rho_{i}$ is compared with the maximum detectable angle denoted by $\theta$. Generally, $\theta$ is determined by the included angle of the stylus flank. In some cases, it is desired that $\theta$ be smaller than this value, such as twothirds of $\theta$, to avoid the phenomenon of stylus jumping. If $\rho_{i}$ is in the one degree tolerance range of $\theta$, it is reasonable to conclude that the contact point, $C_{i}$, moved to the stylus flank. In this case, point $C_{i}^{\prime}$, which was calculated by a stylus radius correction algorithm, would deviate from the real sample surface, as shown in Figure 4(b). As $C_{i}^{\prime}$ is used in the measurement to represent the sample surface, profile distortion would be generated.

In this case, the $X$ motorized driven stage with the sample would be tilted by the spindle at a preset step angle $\beta$. $\beta$ is set to be positive along the anticlockwise direction and negative along the clockwise direction. The previous scanning results, therefore, follow the rotation, and their updated coordinates can be recalculated by

$$
\left[\begin{array}{c}
P_{1}^{\prime} \\
P_{2}^{\prime} \\
P_{3}^{\prime} \\
\vdots \\
P_{i}^{\prime}
\end{array}\right]=\left[\begin{array}{cc}
P_{1-X}^{\prime} & P_{1-z}^{\prime} \\
P_{2-X}^{\prime} & P_{2-Z}^{\prime} \\
P_{3-X}^{\prime} & P_{3-Z}^{\prime} \\
\vdots & \vdots \\
P_{i-X}^{\prime} & P_{i-Z}^{\prime}
\end{array}\right] \times\left[\begin{array}{cc}
\cos \beta & \sin \beta \\
-\sin \beta & \cos \beta
\end{array}\right] .
$$




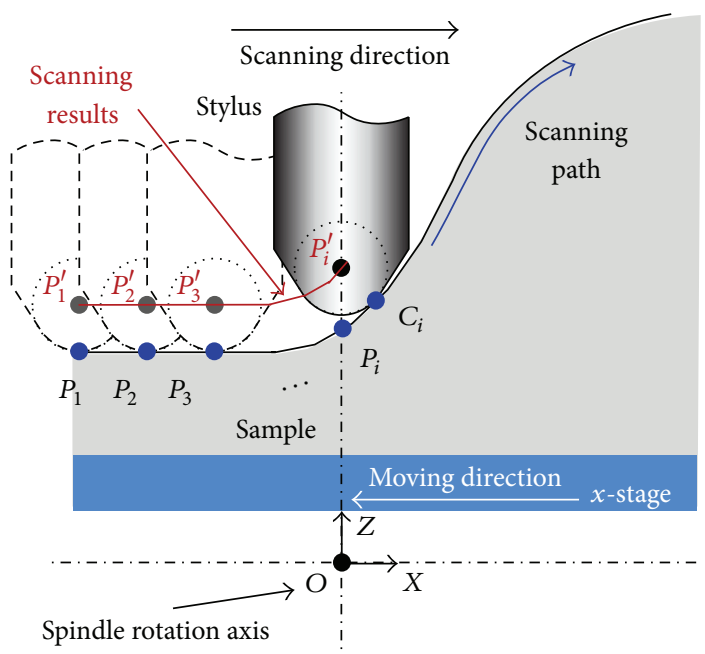

(a)

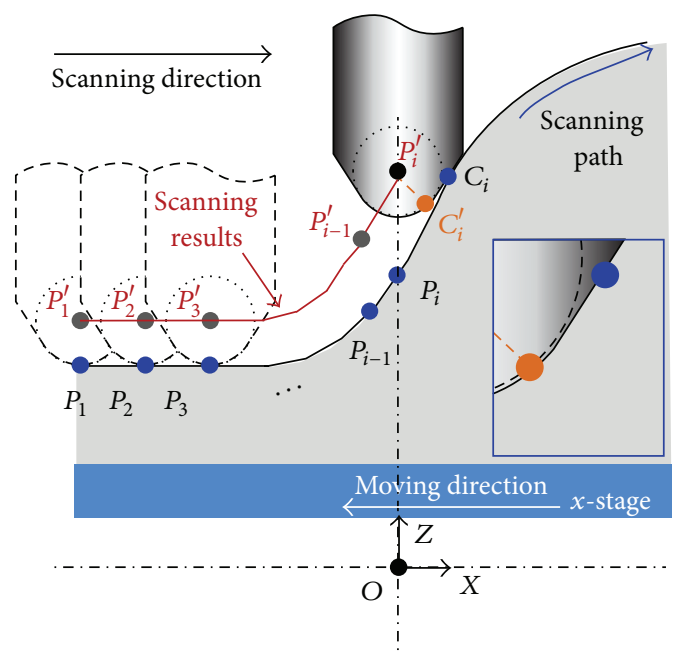

(b)

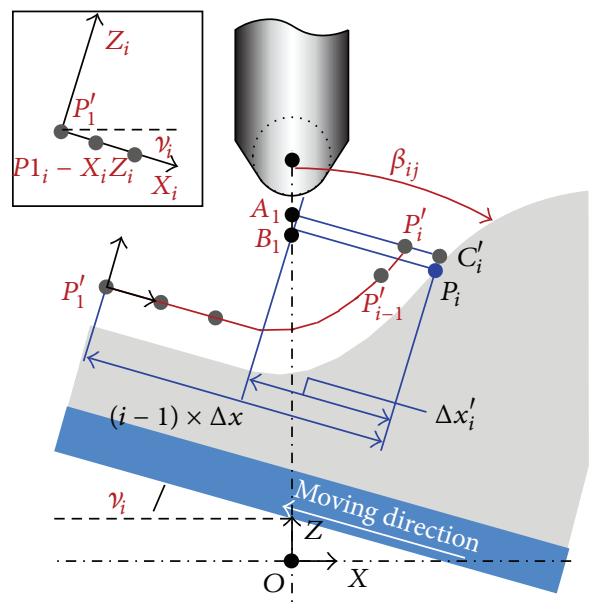

(c)

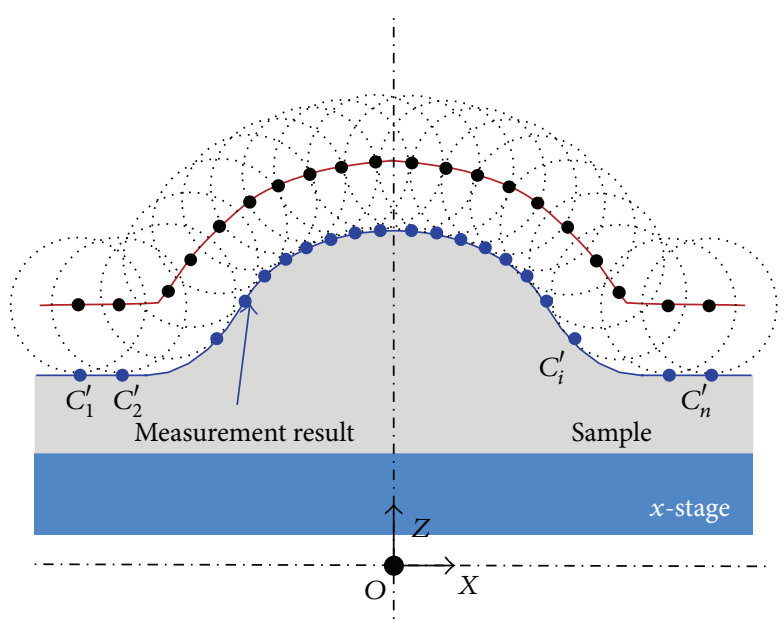

(d)

FIGURE 4: Control methods of the sample-tilting and the $X$ motorized stage. (a) Schematic of stylus scanning. (b) Principle of profile deviation. (c) Schematic of sample-tilting. (d) Schematic of profile reconstruction.

After the rotation, the current measuring point, $P_{i}$, is not located at the ideal position where the $x$-coordinate is zero, which was shown in Figure 4(c). Because the exact spatial coordinates of $P_{i}$ are unknown, $P_{i}$ cannot be easily corrected to the ideal position by the $X$ stage moving a given distance. The last three points $\left[\begin{array}{lll}P_{i-2}^{\prime} & P_{i-1}^{\prime} & P_{i}^{\prime}\end{array}\right]$ of the scanning results after the coordinate transformation are used to estimate the current spatial coordinates of $P_{i}$. The slope of the curve, which is obtained by the first-order linear fitting of the points $\left[\begin{array}{lll}P_{i-2}^{\prime} & P_{i-1}^{\prime} & P_{i}^{\prime}\end{array}\right]$, is denoted by $\rho_{i}^{\prime}$. The current coordinates $\left[\begin{array}{ll}P_{i-X} & P_{i-Z}\end{array}\right]$ of $P_{i}$ are expressed as

$$
\left[\begin{array}{ll}
P_{i-X} & P_{i-Z}
\end{array}\right]=\left[\begin{array}{ll}
P_{i-X}^{\prime}+r \cdot \cos \rho_{i}^{\prime} & \left.P_{i-Z}^{\prime}-r \cdot\left|\sin \rho_{i}^{\prime}\right|\right] .
\end{array}\right]
$$

The total rotation angle of the spindle until this point is denoted by $v_{i}$, which is the cumulative sum of $\beta$. The displacement of the $X$ motorized driven stage is denoted by $\Delta x_{i}^{\prime}$ and derived from

$$
\Delta x_{i}^{\prime}=\frac{P_{i-X}}{\cos v_{i}} .
$$

After the position of $P_{i}$ along the $X$-direction is corrected, $P_{i}$ can be measured and the local slope $\rho_{i}$ is detected again. The sample-tilting process is repeated until the local slope $\rho_{i}$ is smaller than $\theta$. If $\rho_{i}$ meets the requirement relative to $\theta$, the surface scanning turns to $P_{i+1}$, and the moving distance of the $X$ motorized stage is expressed as

$$
\Delta x_{i+1}=\frac{\Delta x}{\cos v_{i}} .
$$

It should be noted that the cumulative rotation angle of the sample-tilting is limited by the maximum rotation angle 
of the spindle, denoted by $\varphi$, which is manually set according to the motion error of the spindle.

After the surface scanning is finished, the orientation of the scanning results can be transformed to its initial state by

$$
\begin{aligned}
{\left[\begin{array}{c}
P_{1}^{\prime} \\
P_{2}^{\prime} \\
P_{3}^{\prime} \\
\vdots \\
P_{N}^{\prime}
\end{array}\right]=} & {\left[\begin{array}{cc}
P_{1-X}^{\prime} & P_{1-Z}^{\prime} \\
P_{2-X}^{\prime} & P_{2-Z}^{\prime} \\
P_{3-X}^{\prime} & P_{3-Z}^{\prime} \\
\vdots & \vdots \\
P_{N-X}^{\prime} & P_{N-Z}^{\prime}
\end{array}\right] } \\
& \times\left[\begin{array}{cc}
\cos \left(-v_{N}\right) & \sin \left(-v_{N}\right) \\
-\sin \left(-v_{N}\right) & \cos \left(-v_{N}\right)
\end{array}\right]
\end{aligned}
$$

where $v_{N}$ is the total rotation angle after the last point $P_{N}$ is measured and $N$ is the number of sampling points.

As shown in Figure 4(d), the scanning result is an envelope of the sample surface. A stylus tip radius correction algorithm based on a first-order linear fitting method was used to eliminate the error introduced by the flank of the stylus tip. Assume that $\rho_{i}^{\prime \prime}$, which is obtained by the first-order linear fitting method, is the slope of each point $P_{i}^{\prime}$. The contact point $C_{i}^{\prime}$, the measurement results to represent the sample surface, can be obtained from

$$
\begin{aligned}
& {\left[\begin{array}{c}
C_{1}^{\prime} \\
C_{2}^{\prime} \\
C_{3}^{\prime} \\
\vdots \\
C_{N}^{\prime}
\end{array}\right]} \\
& =\left[\begin{array}{cc}
C_{1-X}^{\prime} & C_{1-z}^{\prime} \\
C_{2-X}^{\prime} & C_{2-Z}^{\prime} \\
C_{3-X}^{\prime} & C_{3-Z}^{\prime} \\
\vdots & \vdots \\
C_{N-X}^{\prime} & C_{N-Z}^{\prime}
\end{array}\right] \\
& =\left[\begin{array}{cc}
P_{1-X}^{\prime}+r \cdot(k) \cdot \cos \rho_{1}^{\prime \prime} & P_{1-z}^{\prime}-r \cdot\left|\sin \rho_{1}^{\prime \prime}\right| \\
P_{2-X}^{\prime}+r \cdot(k) \cdot \cos \rho_{2}^{\prime \prime} & P_{2-Z}^{\prime}-r \cdot\left|\sin \rho_{2}^{\prime \prime}\right| \\
P_{3-X}^{\prime}+r \cdot(k) \cdot \cos \rho_{3}^{\prime \prime} & P_{3-Z}^{\prime}-r \cdot\left|\sin \rho_{3}^{\prime \prime}\right| \\
\vdots & \vdots \\
P_{N-X}^{\prime}+r \cdot(k) \cdot \cos \rho_{N}^{\prime \prime} & P_{N-Z}^{\prime}-r \cdot\left|\sin \rho_{N}^{\prime \prime}\right|
\end{array}\right]
\end{aligned}
$$

In (7), the range of $\rho_{i}^{\prime \prime}$ is from $-\pi / 2$ to $+\pi / 2$, and if $\rho_{i}^{\prime \prime}$ is equal to or greater than zero, the coefficient $k$ is 1 ; otherwise, it is -1 .

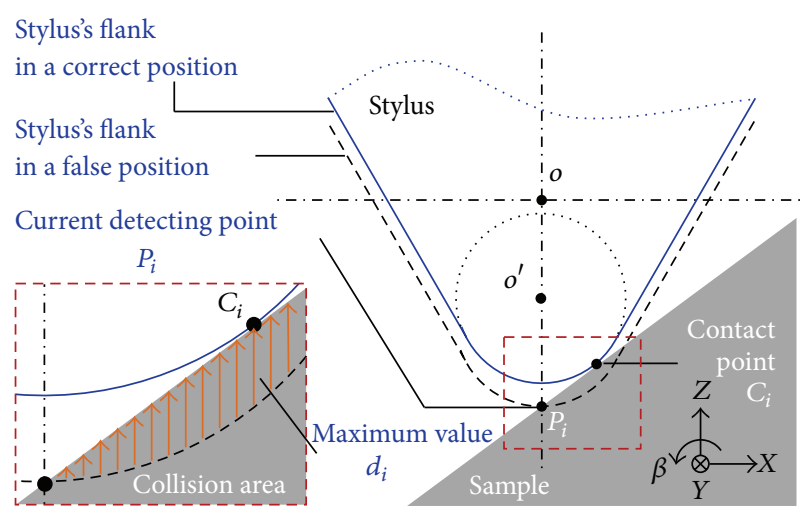

FIGURE 5: Schematic of detecting the contact point.

\section{Simulation Method of the Surface Profile Measurement}

To verify the feasibility of the proposed method, MATLAB was used to carry out the surface profile measurement of microstructures with steep surfaces. The surfaces of the employed stylus and the microstructure under test are dispersed to be point clouds with equal intervals in the $X$ direction. The motion of the stylus is simulated by modifying its $z$-coordinates. Therefore, the variation of the $z$ coordinates is the output of the displacement sensor. Similarly, the motion of the $X$ motorized driven stage is carried out by changing the $x$-coordinates of the microstructure. The sample-tilting and data processing are carried by the method described in the previous section. In a traditional profiling method, the motions of the stylus and the $X$ motorized driven stage are used to construct the surface profile of the sample. The proposed method employs a spindle to adjust the samplestylus relative angle. Therefore, the remaining issue is how to simulate the physical contact between the stylus tip and the sample surface.

Figure 5 illuminates the method to detect the contact point between the stylus flank and the sample surface. When measuring a steep surface, the stylus moves depending on the variation of the surface profile of the sample under test. The contact position is not located at the current detecting point $P_{i}$ but at a point nearby. In the simulation, the stylus tip is first moved a distance, which is denoted by $w_{i}$, to $P_{i}$. The collision area is then detected by comparing the $z$-coordinates of the stylus and the sample. The point where the difference in the $z$ coordinates has a maximum value is the correct contact point $C_{i}$, and the maximum difference is denoted by $d_{i}$. Next, the stylus draws back by a distance $d_{i}$, and the correct contact point $C_{i}$ can be determined. The stylus position denoted by $z_{i}$ is stored as the output of the displacement sensor and is expressed as

$$
z_{i}=w_{i}-d_{i}
$$

\section{Measurement Simulation and Analysis}

Two types of microstructures with steep surfaces were employed to verify the feasibility of the slope-adapted 

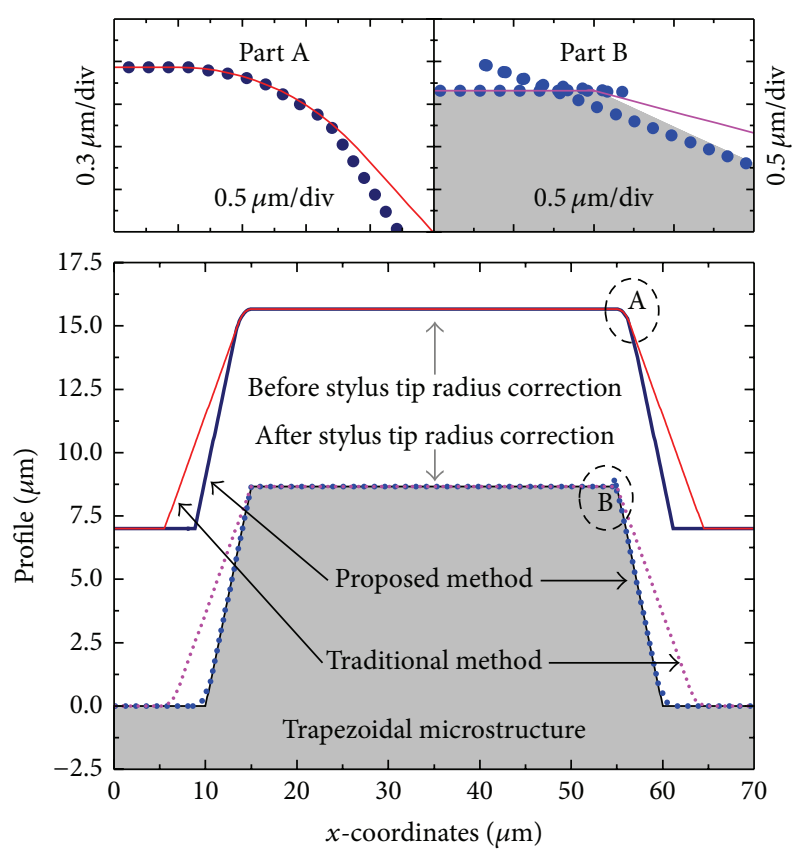

FIgURE 6: Results of profiling a trapezoidal microstructure.

sample-tilting method. The samples were measured twice, using the proposed method and the traditional scanning method, in which the relative direction of the sensing axis of the probing system to the measurement sample surface is unchangeable and the maximum detectable angle is limited by the included angle of the employed stylus [13], to demonstrate the improved measurement capability on steep surfaces.

A trapezoidal microstructure with a maximum local slope of up to 60 degrees was measured first. The height of the microstructure is $8.66 \mu \mathrm{m}$, and its whole width is $70 \mu \mathrm{m}$. A diamond-tipped stylus, which is employed in the hardware realization, was introduced into the simulation. The tip radius of the stylus is two micrometers with an included angle of 90 degrees, which means that the maximum detectable angle is 45 degrees. The interval of the surface discretization was set to $0.01 \mu \mathrm{m}$, and the scanning interval was set to $0.1 \mu \mathrm{m}$. The maximum sample-stylus relative angle was set to 30 degrees, which is $2 / 3$ of the maximum detectable angle, 45 degrees. No maximum rotation angle of the spindle was set, to research the motion feature of the rotating spindle. In addition, the step-angle of the sample-tilting was set to be one degree.

Figure 6 shows the measurement results of the trapezoidal microstructure. Profiles before and after the stylus tip radius correction are plotted. As shown in the figure, the measurement result of the proposed method is much more accurate than that of the traditional method because it is much closer to the real surface of the sample. In contrast, the result of the traditional method shows obvious profile deviation and a blind region on the sample side flanks, where the local slope is up to 60 degrees. The measurement of the proposed method on part " $\mathrm{B}$ " exhibits data confusion, which, upon comparing the data on parts " $\mathrm{A}$ " and "B," was

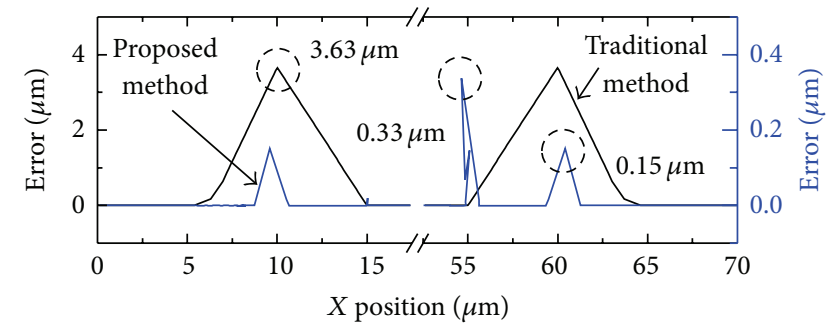

FIGURE 7: Errors in profiling a trapezoidal microstructure.

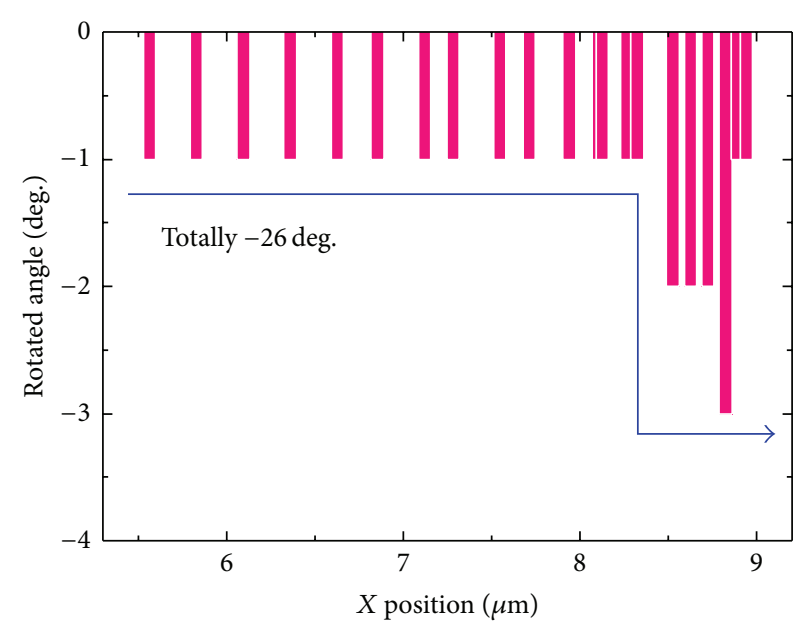

(a)

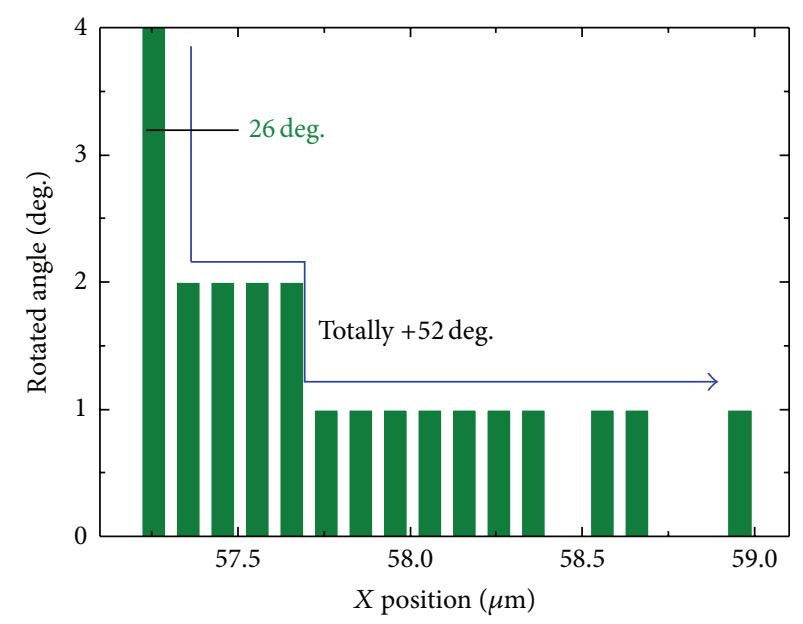

(b)

FIgURE 8: Sample-tilting process of profiling a trapezoidal microstructure.

determined to result from the stylus tip radius correction algorithm.

The measurement errors shown in Figure 7 were calculated by comparing the $y$-coordinates with the real profile of the sample. As shown in the figure, the measurement error of the traditional method was up to $3.63 \mu \mathrm{m}$, while that of the proposed method was reduced to $0.15 \mu \mathrm{m}$. It should be noted that the peak value of $0.33 \mu \mathrm{m}$ in the error curve of the proposed method resulted from the data treatment of the stylus radius correction. Figure 8 shows the sample-tilting 


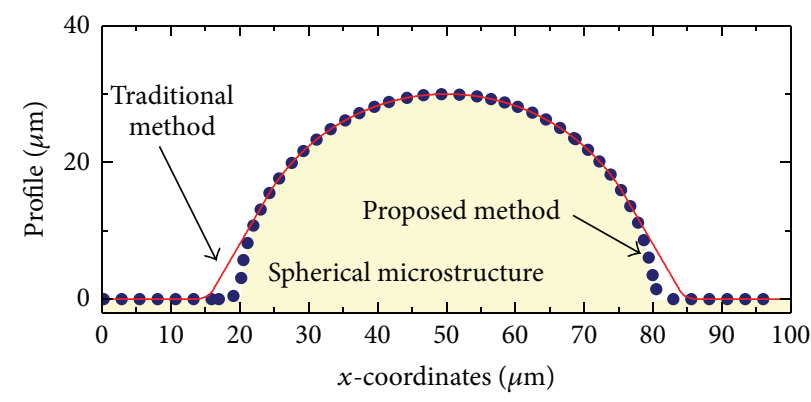

FIgURE 9: Results of profiling a spherical microstructure.

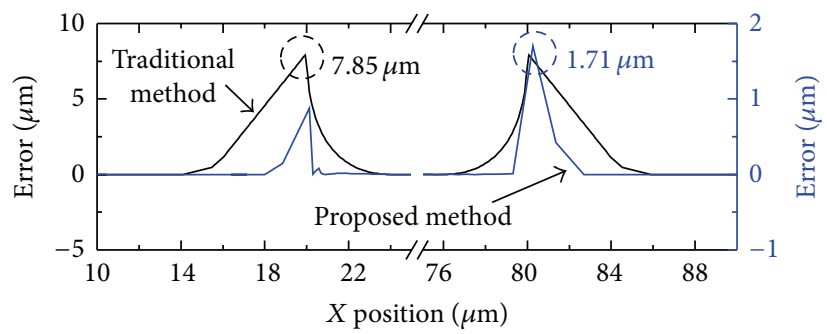

FIGURE 10: Errors in profiling a spherical microstructure.

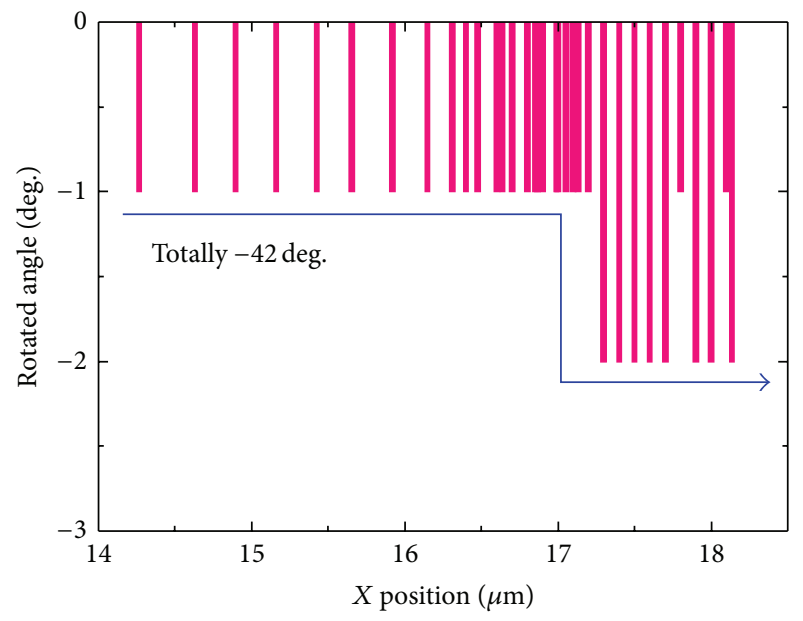

(a)

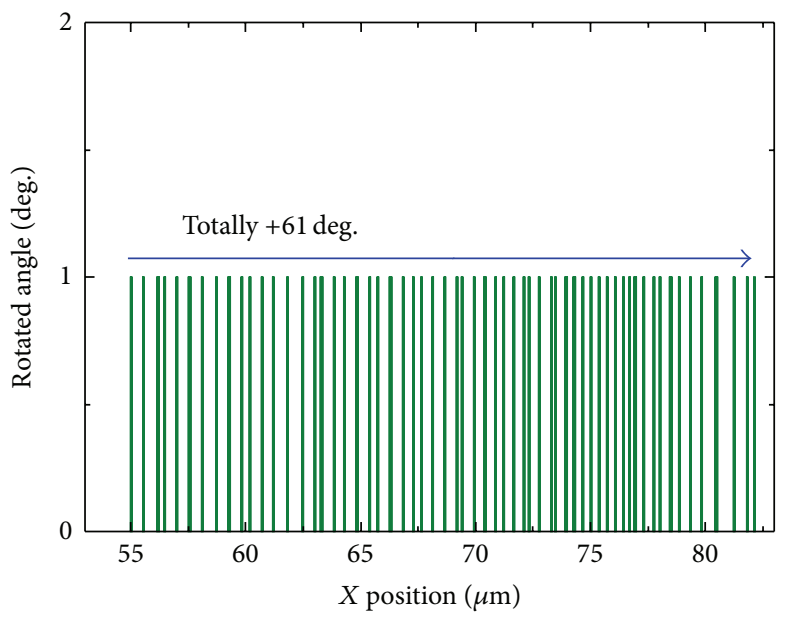

(b)

FIGURE 11: Sample-tilting process of profiling a spherical microstructure.

process. The sample was tilted by a total of 26 degrees in a clockwise direction when measuring the left part of the sample, over the $x$-coordinates range of $5 \mu \mathrm{m}$ to $9 \mu \mathrm{m}$. When measuring the right part, the sample was tilted by a total of 52 degrees in an anticlockwise direction. It should be mentioned that when measuring a position at an $x$ coordinate of $57.25 \mu \mathrm{m}$, the sample is rotated by 26 degrees at once.

A spherical microstructure with a radius of $30 \mu \mathrm{m}$ was employed as the secondary sample. In the measurement, the included angle of the stylus was set to 60 degrees, and the maximum sample-stylus relative angle was set to 45 degrees, which is $3 / 4$ of the maximum detectable angle, 60 degrees. The step-angle of the sample-tilting was set to two degrees.
The other parameters were the same as those used in profiling the trapezoid microstructure.

Figure 9 displays the measurement results of the spherical microstructure. As shown in the figure, the profiles overlapped with each other over most parts of the sample surface, except for the regions around the two concave corners. This result indicates that the proposed method is able to measure a steep surface even with a local slope of up to 90 degrees. Figure 10 compares the measurement errors of the two methods, which were linked to the left and right $y$-axes, respectively. The maximum error of the traditional method is $7.85 \mu \mathrm{m}$ and that of the proposed method is reduced to $1.71 \mu \mathrm{m}$. Figure 11 shows the sample-tilting process. The sample was tilted by a total of 42 degrees in the clockwise 
direction when measuring the left part and by a total of 61 degrees in the anticlockwise direction for the right part.

\section{Conclusion}

A slope-adapted sample-tilting method has been proposed for the profile measurement of microstructures with steep surfaces. Theoretical work required to realize a system to implement the proposed method has been completed. Profile measurements of trapezoidal and spherical microstructures have been simulated to verify the performance of the developed method. The measurement results reveal that the steep surface of a microstructure can be precisely measured by the developed method.

The future work of this research will focus on analyzing the influence of the spindle motion error and realizing the stylus-based profiling system.

\section{Conflict of Interests}

The authors declare that there is no conflict of interests regarding the publication of this paper.

\section{Acknowledgments}

This work is supported by the National Natural Science Foundation of China (Grant no. 51405314) and by the Fundamental Research Funds for the Central Universities (Grant no. 2015SCU04A12).

\section{References}

[1] A. Weckenmann, G. Peggs, and J. Hoffmann, "Probing systems for dimensional micro- and nano-metrology," Measurement Science and Technology, vol. 17, no. 3, pp. 504-509, 2006.

[2] P. M. Lonardo, D. A. Lucca, and L. De Chiffre, "Emerging trends in surface metrology," CIRP Annals-Manufacturing Technology, vol. 51, no. 2, pp. 701-723, 2002.

[3] M. B. Bauza, S. C. Woody, B. A. Woody, and S. T. Smith, "Surface profilometry of high aspect ratio features," Wear, vol. 271, no. 34, pp. 519-522, 2011.

[4] H. N. Hansen, K. Carneiro, H. Haitjema, and L. de Chiffre, "Dimensional micro and nano metrology," CIRP AnnalsManufacturing Technology, vol. 55, no. 2, pp. 721-743, 2006.

[5] B. Xu, Y. Shimizu, S. Ito, and W. Gao, "A micro-ball tapping probe for form measurement of micro-structured surface," Journal of the Chinese Society of Mechanical Engineers, vol. 34, no. 1, pp. 73-80, 2013.

[6] B. Xu, Z. Jia, X. Li et al., "Surface form metrology of microoptics," in International Conference on Optics in Precision Engineering and Nanotechnology (icOPEN2013), vol. 8769 of Proceedings of SPIE, April 2013.

[7] B. Xu, Y. Shimizu, T. Takeishi, S. Ito, and W. Gao, "Surface form measurement and analysis of a cylindrical workpiece with microstructures," Journal of Advanced Mechanical Design, Systems and Manufacturing, vol. 6, no. 6, pp. 936-948, 2012.

[8] F. Z. Fang, X. D. Zhang, A. Weckenmann, G. X. Zhang, and C. Evans, "Manufacturing and measurement of freeform optics,"
CIRP Annals-Manufacturing Technology, vol. 62, no. 2, pp. 823-846, 2013.

[9] K.-W. Lee, Y.-J. Noh, W. Gao et al., "Experimental investigation of an air-bearing displacement sensor for on-machine surface form measurement of micro-structures," International Journal of Precision Engineering and Manufacturing, vol. 12, no. 4, pp. 671-678, 2011.

[10] K. Takamasu, "Present problems in coordinate metrology for nano and micro scale measurements," MAPAN, vol. 26, no. 1 , pp. 3-14, 2011.

[11] Y. Takaya, M. Michihata, T. Hayashi, and T. Washitani, "Dimensional measurement of microform with high aspect ratio using an optically controlled particle with standing wave scale sensing," CIRP Annals-Manufacturing Technology, vol. 61, no. 1, pp. 479-482, 2012.

[12] B.-F. Ju, Y.-L. Chen, W. Zhang, W. Zhu, C. Jin, and F. Z. Fang, "Note: long range and accurate measurement of deep trench microstructures by a specialized scanning tunneling microscope," Review of Scientific Instruments, vol. 83, no. 5, Article ID 056106, 2012.

[13] B.-F. Ju, Y.-L. Chen, W. Zhang, and F. Z. Fang, "Rapid measurement of a high step microstructure with 90 degrees steep sidewall," Review of Scientific Instruments, vol. 83, no. 1, Article ID 013706, 2012.

[14] F. Marinello, P. Bariani, A. Pasquini, L. De Chiffre, M. Bossard, and G. B. Picotto, "Increase of maximum detectable slope with optical profilers, through controlled tilting and image processing," Measurement Science and Technology, vol. 18, no. 2, pp. 384-389, 2007.

[15] S.-J. Cho, B.-W. Ahn, J. Kim et al., “Three-dimensional imaging of undercut and sidewall structures by atomic force microscopy," Review of Scientific Instruments, vol. 82, no. 2, Article ID 023707, 2011.

[16] S. P. Pan, H. C. Liou, C. C. A. Chen, J.-R. Chen, and T.-S. Liu, "Precision measurement of sub-50 nm linewidth by stitching double-tilt images," Japanese Journal of Applied Physics, vol. 49, no. 6, Article ID 06GK06, 2010.

[17] R. Henselmans, L. A. Cacace, G. F. Y. Kramer, P. C. J. N. Rosielle, and M. Steinbuch, "The NANOMEFOS non-contact measurement machine for freeform optics," Precision Engineering, vol. 35, no. 4, pp. 607-624, 2011.

[18] A. Schuler and A. Weckenmann, "Application of sensor tilting for enhanced measurement of microstructures," Proceedings of the Institution of Mechanical Engineers, Part B: Journal of Engineering Manufacture, vol. 227, no. 1, pp. 12-20, 2013.

[19] A. Weckenmann, A. Schuler, and R. J. B. Ngassam, "Enhanced measurement of steep surfaces by slope-adapted sensor tilting," Measurement Science and Technology, vol. 23, no. 7, Article ID 074007, 2012.

[20] J. W. Cui, L. Li, J. Y. Li, and J. B. Tan, "Fiber probe for microhole measurement based on detection of returning light energy," Sensors and Actuators A: Physical, vol. 190, pp. 13-18, 2013.

[21] S. Z. Wang, T. B. Xie, and S. P. Chang, "A white light interference-based atomic force probe scanning microscopy," Measurement Science and Technology, vol. 22, no. 4, Article ID 045502, 2011.

[22] J. A. Kramar, R. Dixson, and N. G. Orji, "Scanning probe microscope dimensional metrology at NIST," Measurement Science and Technology, vol. 22, no. 2, Article ID 024001, 2011.

[23] W. Gao, B. Xu, T. Takeishi, Y. Shimizu, and S. Ito, "Selfcalibration and compensation of setting errors for surface 
profile measurement of a microstructured roll workpiece," Chinese Journal of Mechanical Engineering, vol. 27, no. 1, pp. 1422, 2014.

[24] B. Xu, Y. Shimizu, S. Ito, and W. Gao, "Surface profile measurement of internal micro-structures," International Journal of Precision Engineering and Manufacturing, vol. 14, no. 9, pp. 1535-1541, 2013. 

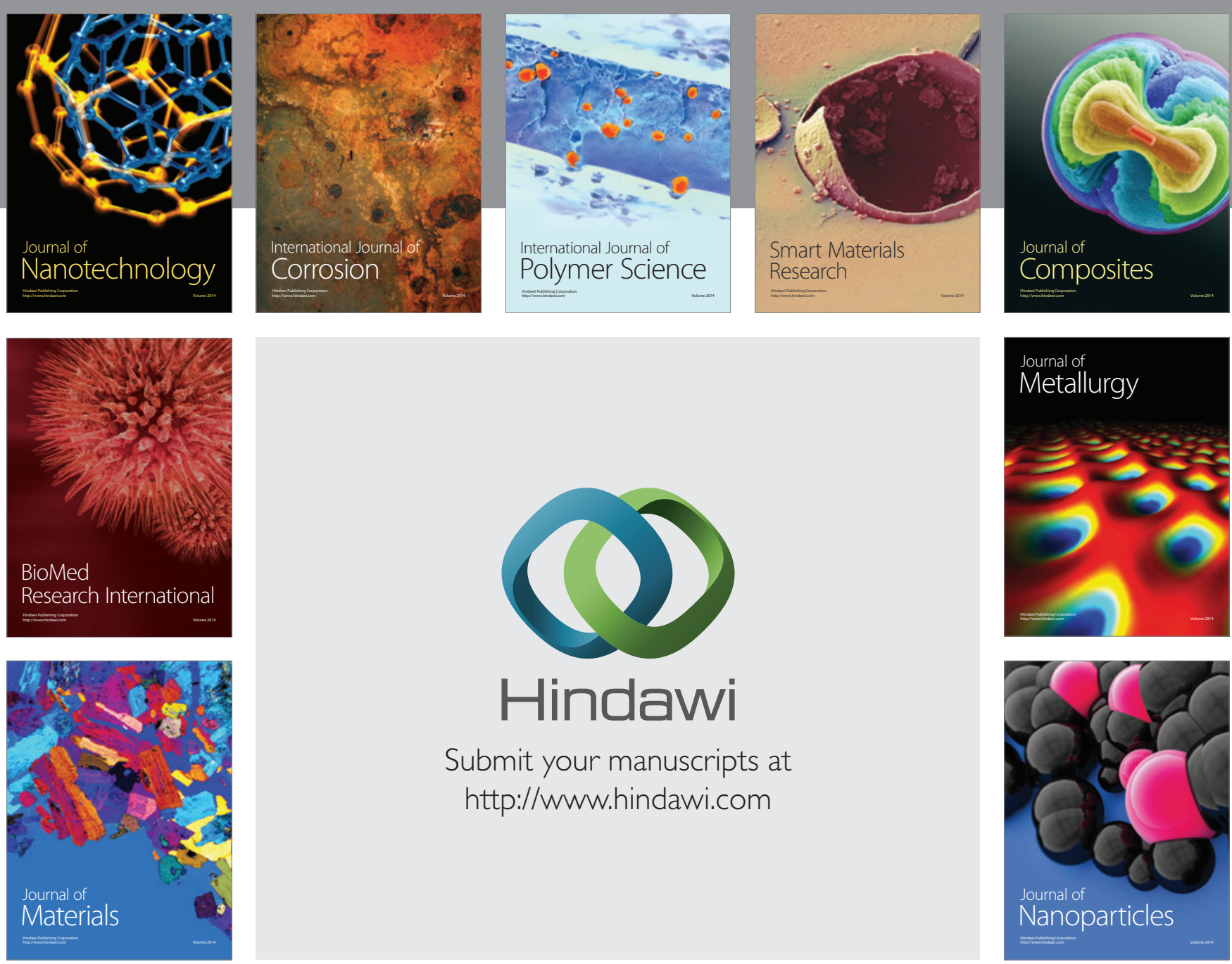

Submit your manuscripts at http://www.hindawi.com
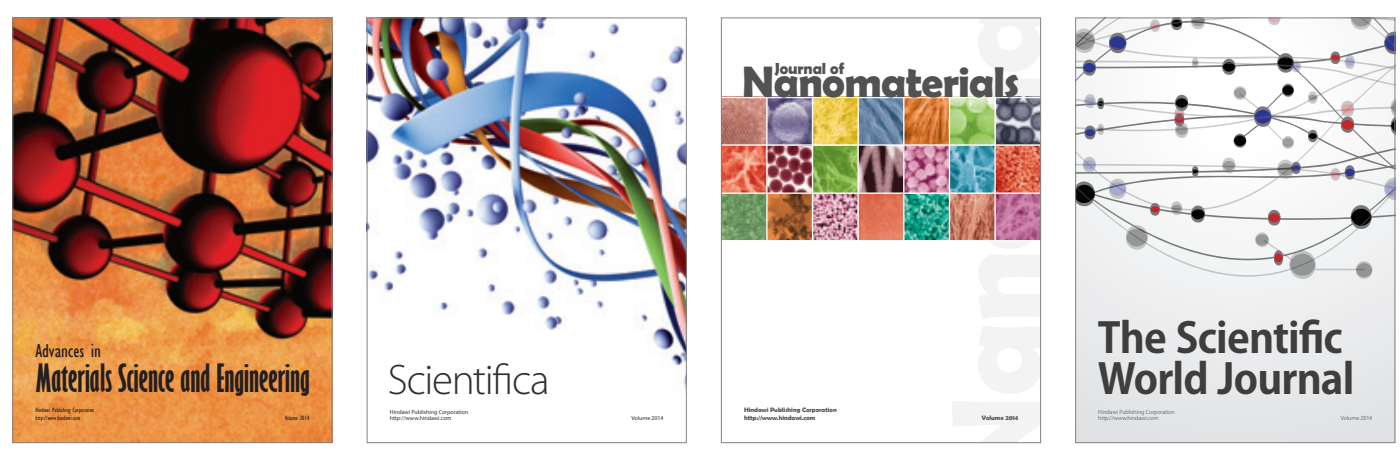

\section{The Scientific World Journal}
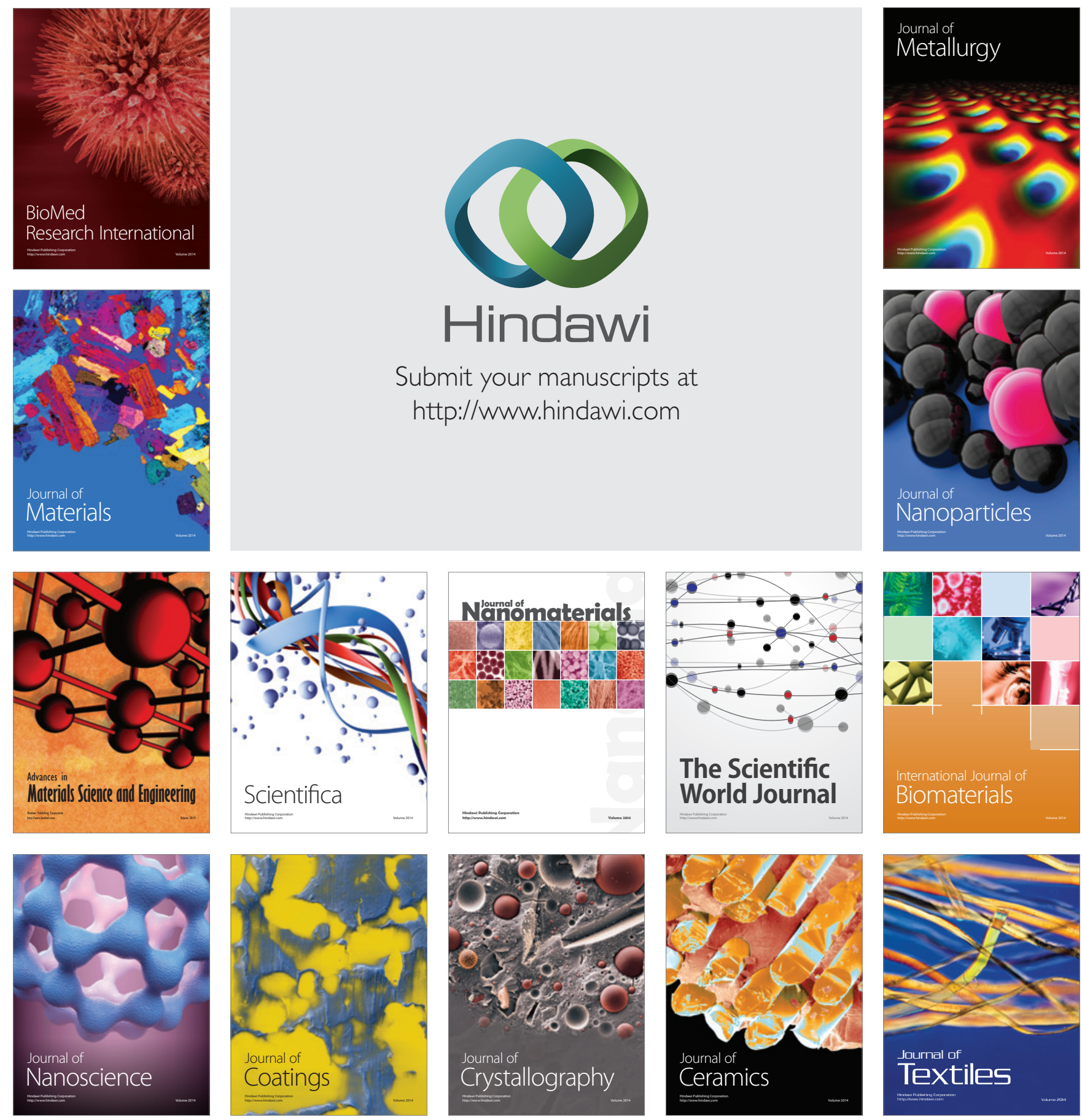